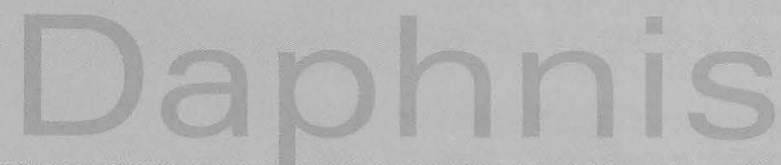

\title{
Zeitechrift für
}

Mittlere Deutsche Literatur und Kultur der Frühen Neuzelt

$[1400 \cdot 1750]$

BAND 31 - 2002

HEFT $3=4$

\section{Herausgegeben von}

Barbara Eecker-Cantarino - Martin Bireher Mirastawa Czannecka - Kaus Earber Ferdinand van Ingen - Knut Kiesant Wilhelm Kühimann - Eberhard Mannaek

Abarto Martino - Wolfoang Neuber Hang-Cart Foloff - Ulrieh Eeelbach Blake Lee Gpahr - Jean-Marie Valentin Helen Watanabe-O'Kelly 
"ET RESPONDEAT". Studien zum deutschen Theater des Mittelalters. Hrsg. von Katja Scheel. - Leuven: Leuven University Press 2002. (= Mediaevalia Lovaniensia. Series I. Studia 32.) XI, 266 S. $€$ 33,-

„Et respondeat“. Diese aus alten Spielen bekannte Regieanweisung fungiert hier als Titel einer Festschrift, die dem Löwener Germanisten Johan Nowé anlässlich seiner Emeritierung gewidmet ist. Nowé trat mehrfach als Kenner des mittelalterlichen Theaters hervor, zuletzt im Jahr 2000 mit dem Erscheinen seines Buches $N u$ hoort wat men $u$ spelen zal. Theater in de Middeleeuwen (Leuven: Davidsfonds/Literair), sowie mit einer Inszenierung des Redentiner Osterspiels (vgl. http://redentiner.osterspiel.org/) und der Organisation des wissenschaftlichen Kolloquiums "Das religiöse Drama des deutschen Mittelalters" in Löwen (vgl. Leuvense Bijdragen 90, 2001, H. 13). Auf diesem Forschungsfeld des Jubilars bewegen sich auch die zwölf Beiträge der Festschrift, die in einem großzügigen Verständnis von 'Mittelalter' die Dramen des 16. Jahrhunderts mit einbeziehen.

Der inhaltliche Schwerpunkt des Bandes liegt auf dem geistlichen Spiel, dem sich die ersten Aufsätze werkübergreifend mit verschiedenen methodischen Ansätzen nähern. So stellt Luc De Grauwe semasiologisch den Wortgebrauch von 'Trost' und 'Tröster' in Spielen und verwandten Gattungen dar, speziell in Bezug auf Christus als 'Consolator' (S. 1-27). Cobie Kuné untersucht ikonographisch die Wechselbeziehungen zwischen Drama und bildender Kunst am Beispiel von Christi Gebet am Ölberg (S. 29-50, mit Abb. auf S. 28). Die Behandlung des Emmausstoffs in den deutschen Spielen vergleicht Walter De Cubber, der ein besonderes Augenmerk auf mögliche Ausweitungen gegenüber dem Bibeltext richtet (S. 51-85). Eine weitere Gruppe von Studien wendet sich einzelnen Dramen zu, voran dem Redentiner Osterspiel, das Hartmut Freytag, Maike Claußnitzer und Susanne Warda 'sub specie aeternitatis' betrachten, indem sie den heilsgeschichtlichen Elementen in ihm eine gründliche 'Skizze' widmen (S. 87-108). Um einen sprachlichen Einzelaspekt geht es dagegen in der Miszelle "Germanisch [sk] in der Sprache des Redentiner Osterspiels" von Luk Draye (S. 109-111). Von einer Neubearbeitung des Teufel-Seelen-Spiels als Das Teufelsspiel von Redentin durch den DDR-Dramatiker Claus Hammel, die 1987 in Rostock aufgeführt wurde, berichtet Irmtraud Rösler (S. 113130). Die eschatologische Thematik wird in zwei Beiträgen behandelt. Während Hansjürgen Linke das 'iudicium particulare' im Münchner Eigengerichtspiel von 1510 darstellt (S. 131-145), bietet das 'iudicium universale' den Rahmen für den Streit zwischen 'Anima' und 'Corpus' im Weltgerichtsteil des Künzelsauer Fronleichnamspiels, dem ein Aufsatz von Elke Ukena-Best gilt (S. 145-176). Eher als Randphänomen des geistlichen 
Spiels kann der Auftritt eines "Knabenbischofs" ("episcopus puerorum") gelten, der sicher für das Benediktbeurer Weihnachtsspiel nachzuweisen ist, vielleicht auch für das Schwäbische (Konstanzer) Weihnachtsspiel, wo an seiner Stelle freilich ein "Capplan" namens "Biceps" erscheint (wirklich eine Verlesung von "episcopus"?); Michael Straeter ordnet in seinem Beitrag (S. 177-194) diese Figur in die Bräuche des Festes der Schüler und Chorknaben der Weihnachtszeit ein und fragt nach seiner Funktion im mittelalterlichen Schulwesen, dem ja auch ein großer Teil des Theaters zugewiesen werden kann. Aus dem bisher ungedruckten Luzerner Wilhelmspiel von 1596 publiziert John E. Tailby als Teiledition aus dem dritten 'Actus' eine Folterszene, von der er die lateinischen und deutschen Bühnenanweisungen vor allem im Hinblick auf die Bewegungen der Spieler analysiert (S. 195-214). Etwas am Rande der Theaterthematik scheint sich der Beitrag von Leif Søndergaard über "Die Tänze der mittelalterlichen Gilden zur Fastnacht" (S. 215-231, mit Abb. auf S. 232-236) zu bewegen; aber es geht hier vor allem um darstellende Tänze, die vom Autor anhand von schriftlichen und bildlichen Quellen als "Theater ohne Worte" oder "Semi-Theater" (S. 231) gedeutet werden. Mit dem eigentlichen Fastnachtspiel steht im letzten Aufsatz wenigstens ein weltlicher Dramentyp zur Debatte, der von Katja Scheel aus der Genderperspektive betrachtet wird: "Von fremdgehenden Frauen und untreuen Ehemännern: Tugendprobe und Eheproblematik im Fastnachtspiel des 15. Jahrhunderts" (S. 237-258). Drei Register (zu Handschriften, Bibelstellen und zu Namen und Werken) schlieBen den Band ab (S. 259-266).

Die Beiträge bewegen sich allesamt auf hohem Niveau und zeugen von einem erfreulichen Fortschritt, den die Erforschung des vormodernen Theaters, vor allem des geistlichen Spiels, in den letzten Jahren auch in der Germanistik gemacht hat. Obwohl die meisten Studien des Bandes sich philologisch an der Textüberlieferung der Spiele orientieren, gerät doch immer wieder auch deren Aufführungspraxis in den Blick, und zwar speziell durch die Analyse von Regieanweisungen, die der Adressat der Festschrift jüngst mit einer exemplarischen Arbeit zum Redentiner Osterspiel nachdrücklich eingefordert hat (vgl. Johan Nowé: "Wy willen ju eyn bilde gheven". In: Leuvense Bijdragen 90, 2001, S. 325-359). Die Anregungen des Jubilars, insbesondere die Unterscheidung zwischen expliziten auch impliziten Bühnenanweisungen (vgl. S. 212), werden mehrfach fruchtbar gemacht, etwa in dem Versuch, "das Aussehen und das Benehmen Christi beim Gebet" zu rekonstruieren (S. 47), oder in der Untersuchung einer eigenen "Stimmregie" (S. 142). Sehr ergiebig ist dieser Ansatz beim Luzerner Wilhelmspiel, wo sich präzise Folteranweisungen für den Henker finden und analysieren lassen (z.B. S. 206: "Schlizt in", "brent in"). Mit diesem Interesse an den literaturwissenschaftlich bisher meist vernachlässigten Regieanweisungen, auf das auch die Titelgebung verweist, lässt sich den thematisch und methodisch etwas disparaten Beiträgen doch ein roter Faden 
entnehmen. Außerdem ist in dieser Festschrift ein durchgängiges Interesse an intermedialen Aspekten vormoderner Theatralität zu beobachten; so geht es beispielsweise um das Verhältnis von Theater zu Malerei (Kuné), Lehrgespräch (Linke), Liturgie (Freytag u.a.), Festbrauchtum (Straeter) oder Tanz (Søndergaard). Solche Ansätze wären weiter zu verfolgen - in Einzelstudien, vor allem aber in einem systematisierenden Forschungsbericht.

Wuppertal / Bielefeld

Meinolf Schumacher 